\title{
DETERMINAÇÃO DO INTERVALO DE MANUTENÇÃO PROGRAMADA DA PROTEÇÃ̃O DE LINHAS DE TRANSMISSÃO CONSIDERANDO-SE PENALIDADES ASSOCIADAS À INDISPONIBILIDADE
}

\author{
Felipe E. L. Pereira* \\ felipelepuc-rio.br
}

Albert C. G. de Melo ${ }^{\dagger}$

albertecepel.br

\author{
Ricardo B. Prada* \\ prada@ele.puc-rio.br
}

Anselmo Barbosa Rodrigues ${ }^{\ddagger}$

nebulok_99@yahoo.com

\author{
Maria da Guia da Silva $a^{\ddagger}$ \\ guia@dee.ufma.br \\ ${ }^{*}$ Departamento de Engenharia Elétrica, PUC-Rio, \\ Rua Marquês de São Vicente, 225, \\ CEP 22453-900, Rio de Janeiro, RJ \\ ${ }^{\dagger}$ CEPEL, \\ Av. Hum, S/N, Ilha da Cidade Universitária, \\ CEP 2194-590 - Rio de Janeiro, RJ \\ ${ }^{\ddagger}$ Departamento de Engenharia Elétrica, UFMA, \\ Av. dos Portugueses, S/N, \\ CEP 65080-040, São Luís, MA
}

\section{RESUMO}

Determination of Scheduled Maintenance Interval in the Protection of Transmission Lines Considering the Penalties Associated to Transmission Equipament Unavailabilities.

Currently, the National Agency for Electric Energy applies penalties to the transmission companies due to scheduled and non-scheduled outages. These penalties have as objective to assure the reliability of the transmission service in a competitive environment. This paper proposes a method to minimize the penalties associated to the transmission equipment outages through the optimization of the scheduled maintenance

Artigo submetido em 14/05/2010 (Id.: 01149)

Revisado em 07/08/2010, 21/10/2010

Aceito sob recomendação do Editor Associado Prof. Julio Cesar Stacchini Souza interval. The proposed optimization model is based on a new analytic method that estimates the expected penalties accurately.

Keywords: Reliability, Maintenance, Markovian Models, Monte Carlo Simulation, Optimization.

\section{RESUMO}

Atualmente, a ANEEL (Agência Nacional de Energia Elétrica) aplica penalidades nas empresas de transmissão devido aos desligamentos programados e não-programados. $\mathrm{O}$ uso destas penalidades tem como objetivo assegurar a confiabilidade do serviço de transmissão em um ambiente competitivo. Este artigo propõe um método para minimizar as penalidades associadas com desligamentos de equipamentos de transmissão através da otimização dos intervalos das manutenções programadas. O modelo de otimização proposto neste artigo 
se baseia em novo método analítico que é capaz de estimar precisamente os valores esperados das penalidades.

Palavras Chaves - Confiabilidade, Manutenção, Modelos Markovianos, Simulação Monte Carlo, Otimização.

\section{INTRODUÇÃO}

O modelo econômico do setor elétrico brasileiro baseia-se na desagregação entre os agentes de geração, transmissão, distribuição e comercialização de energia. A separação das atividades de geração e transporte de energia elétrica proporcionou a realização de leilões de concessões de linhas de transmissão e a participação de empresas transmissoras ou consórcios nestes leilões. As concessões das linhas de transmissão são dadas para as empresas de transmissão ou consórcios, que ofertarem as menores tarifas de transmissão (menores receitas anuais), desde que os valores ofertados pelos demais proponentes, sejam superiores a um determinado percentual estabelecido para o leilão. Caso seja inferior ao valor do percentual estipulado, o leilão continua sendo realizado através de lances sucessivos por meio de deságios. Devido às suas naturezas, os investimentos em linhas de transmissão são caracterizados por custos de implantação relativamente baixos e por uma receita operacional pré-estabelecida, de acordo com o lance vencedor do leilão, satisfazendo os limites máximos estipulados pela ANEEL. As empresas de transmissão ou consórcios vencedores firmam um Contrato de Prestação de Serviço de Transmissão (CPST) com o Operador Nacional do Sistema Elétrico (ONS), delegando-lhe o direito de administrar e coordenar o uso da rede básica e, em contrapartida, tem garantido o ressarcimento de seus custos e de seus investimentos remunerados. Os investimentos podem ser considerados de baixo risco, já que a receita associada é garantida através do CPST firmado. Consequentemente, o modelo econômico que norteia a prestação de serviços de transmissão de energia elétrica é fundamentado em uma estrutura reguladora. Ao contrário dos geradores e comercializadores, que têm liberdade para estabelecer seu preço no ambiente competitivo, a renda proveniente da transmissão é estabelecida pela ANEEL, como uma Receita Anual Permitida (RAP) pelo "aluguel" de seus ativos.

Os usuários do sistema (geradores, distribuidores e grandes consumidores) arcam com os investimentos necessários para se conectarem à rede básica e pagam ao ONS uma tarifa pelo uso do sistema. Esta tarifa é fixada de forma a cobrir o conjunto dos contratos do ONS com as empresas de transmissão ou consórcios, mais os custos da operação do sistema.

Uma das atribuições do agente regulador é determinar o desconto da RAP de uma empresa de transmissão em função da indisponibilidade de operação de seus equipamentos. Este desconto é denominado Parcela Variável Referente à Dispo- nibilidade de Instalações da Rede Básica, ou simplesmente Parcela Variável (PV) e tem sido tema de grandes debates entre os agentes transmissores, a ANEEL, o ONS e instituições financeiras que têm investido em empreendimentos de expansão do setor elétrico nacional. As indisponibilidades dos ativos de transmissão podem ocorrer de duas maneiras: de forma voluntária ou programada (manutenções, conveniência operativa, manobras, e outras) e por saídas forçadas ou não programadas (falhas). Neste sentido, a fórmula estabelecida pela ANEEL (2007) nas licitações, para calcular a PV tem a forma geral:

$$
\begin{aligned}
P V & =\frac{P B}{1440 \cdot D}\left(\sum_{i=1}^{N P} g\left(D D P_{i}\right)\right) \\
& +\frac{P B}{1440 \cdot D}\left(\sum_{i=1}^{N O} h\left(D O D_{i}\right)\right)
\end{aligned}
$$

onde:

$P V=$ parcela variável por indisponibilidades de equipamentos;

$P B=$ parcela equivalente ao duodécimo da RAP, associada à plena disponibilidade dos ativos que compõem uma instalação de uma empresa de transmissão de energia;

$g\left(D D P_{i}\right)=K_{p} D D P_{i}$

$h\left(D O D_{i}\right)=\left\{\begin{array}{l}K_{O} D O D_{i}, \text { para } D O D_{i} \leq 300 \\ 300 K_{O}+K_{p}\left(D O D_{i}-300\right), \text { para } D O D_{i}>300\end{array}\right.$

$K_{p}$ fator para desligamentos programados, sendo $K_{p}=10$;

$K_{o}$ fator para desligamentos não programados, sendo $K_{o}=$ 150 ;

$N P$ número de desligamentos programados da instalação durante o mês;

NO número de desligamentos não programados da instalação ao longo do mês;

$D$ número de dias do mês;

$D D P_{i}$ duração em minutos de cada desligamento programado que ocorra no mês;

$D O D_{i}$ duração em minutos de cada desligamento não programado que ocorra no mês.

A partir de (1) percebe-se que a PV é uma variável aleatória, ou seja, depende das indisponibilidades programadas (manutenção preventiva) e não programadas. Por sua vez, estas últimas, dependem das durações das falhas dos ativos de transmissão, a saber: falhas permanentes (queda de torres, 
falha de equipamento, falha humana, etc.), onde é necessária a intervenção da equipe de manutenção, e falhas transitórias (descarga atmosférica, queimadas), onde o ativo retorna à operação pela atuação de religamento manual ou automático. Neste contexto, torna-se evidente que as empresas de transmissão devem reduzir o custo decorrente da PV, de modo a maximizar o seu lucro anual. Uma das principais variáveis associadas com a minimização da PV é o intervalo de manutenção. Contudo, esta variável tem efeitos conflitantes sobre a PV. As atividades de manutenção melhoram a condição dos equipamentos. Consequentemente é possível minimizar as penalidades devido aos desligamentos não-programados reduzindo os intervalos de manutenção. Por outro lado, o aumento na frequência de manutenções causa uma elevação nas penalidades devido aos desligamentos programados. Devido a isto, as empresas de transmissão devem determinar os intervalos de manutenção visando obter um equilíbrio entre as penalidades causadas por desligamentos programados e não programados. Desta forma, o principal objetivo deste artigo é apresentar um método que minimize a PV através do ajuste do intervalo de manutenção das proteções das linhas de transmissão. A principal característica do modelo de otimização proposto neste artigo é o estabelecimento de um compromisso aceitável entre as parcelas da PV associadas com desligamentos programados e não-programados. Adicionalmente, o modelo de otimização da PV utiliza um método analítico que pode estimar precisamente o valor esperado da PV. Os resultados dos testes demonstraram que as soluções geradas pelo algoritmo de minimização da PV são melhores que aquelas obtidas via maximização da disponibilidade (Anders, 1990). Além disso, as estimativas da PV mostradas neste artigo são mais precisas que aquelas calculadas com base nos método analíticos de estimação do custo de interrupção (Billinton \& Allan, 1996). O restante do artigo é organizado da seguinte forma:

i) na Seção 2 apresenta-se o modelo Markoviano usado para modelar a indisponibilidade das linhas de transmissão;

ii) o método analítico de estimação da PV é apresentado na Seção 3;

iii) na Seção 4 descreve-se o modelo de otimização da PV; $\mathrm{e}$

iv) os resultados e as conclusões são apresentados nas Seções 5 e 6.

\section{MODELO MARKOVIANO PARA EQUI- PAMENTOS DE PROTEÇÃO DE LINHAS DE TRANSMISSÃO}

Os modos de falha de equipamentos de proteção do sistema elétrico foram definidos por Siqueira (1999). A partir da aná- lise dos modos de falhas, é possível identificar o conjunto dos estados possíveis de um sistema de proteção. Inicialmente, três estados são identificados, quando a proteção estiver disponível para a operação:

i) Normal: se a proteção estiver apta a executar sua missão, atuando conforme projetada. Em outras palavras, não apresentar defeito interno ou intrínseco, estando em condições de desligar o equipamento protegido na ocorrência de um defeito interno do mesmo e, simultaneamente, bloquear seu desligamento na ausência de defeito ou na ocorrência de defeitos externos ao processo.

ii) Falha causada por desligamento indevido do sistema de proteção: quando a proteção desligar intempestivamente o equipamento protegido, na ausência de uma falha no equipamento protegido, necessitando de reparo para voltar a operar (manutenção corretiva).

iii) Falha causada por recusa de atuação do sistema de proteção: é o caso onde a proteção apresenta uma falha que a impeça de atuar na ocorrência de uma falha no equipamento protegido. A proteção voltará ao estado normal após a realização de manutenção corretiva. Neste caso, o equipamento protegido será desligado pelas proteções de retaguarda de outros equipamentos, ou pelos sistemas de proteção contra falha de disjuntor, em consequência da recusa de atuação da proteção. Neste caso, o equipamento também exige a realização de uma manutenção corretiva.

iv) Teste: quando a proteção estiver sendo submetida a uma inspeção ou teste periódico, manutenção preventiva ou auto-teste.

O diagrama de Markov usado para representar as transições entre os quatro estados de operação definidos é mostrado na Figura 1 (Pereira, 2009), onde:

$\lambda_{12}$ taxa de transição entre os estados normal e teste;

$\mu_{21}$ taxa de transição entre os estados teste e normal;

$\lambda_{13}$ taxa de falha do estado normal para falha por desligamento indevido;

$\mu_{31}$ taxa de reparo do estado desligamento indevido para normal;

$\lambda_{14}$ taxa de falha do estado normal para falha causada por recusa;

$\mu_{41}$ taxa de reparo do estado falha causada por recusa para normal. 
No sistema em estudo, agrupam-se os estados de falha devido à recusa de atuação do sistema de proteção e falha devido a desligamentos em um único estado não-conforme, doravante denominado de falha. Para manter a ocorrência lógica, o agrupamento só será viável se o novo modelo permanecer um sistema Markoviano. Para isto será suficiente que as taxas de transição do estado agregado para qualquer outro estado, permaneçam iguais às da cadeia original (Howard, 1971; Endrenyi, 1978). Os valores das probabilidades calculadas após agrupamento dos estados só são precisos em regime permanente, ou seja, o agrupamento de estados não pode ser usado quando se deseja calcular valores transitórios de probabilidade (Endrenyi, 1978). A Figura 2 (Pereira, 2009), ilustra o diagrama de transição entre estados do processo de Markov resultante, no qual o estado agregado é representado pelo número cinco, os demais estados permanecem com a numeração original.

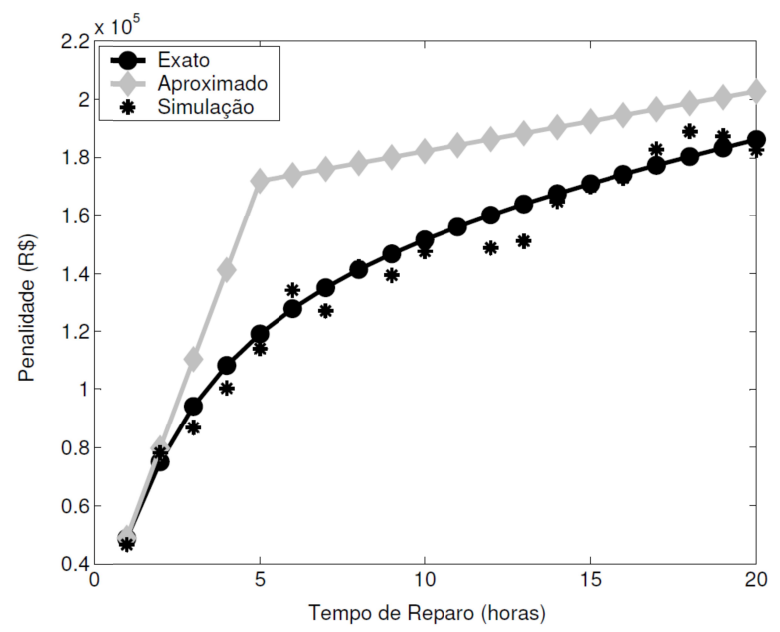

Figura 1: Modelo de Markov para o sistema de proteção

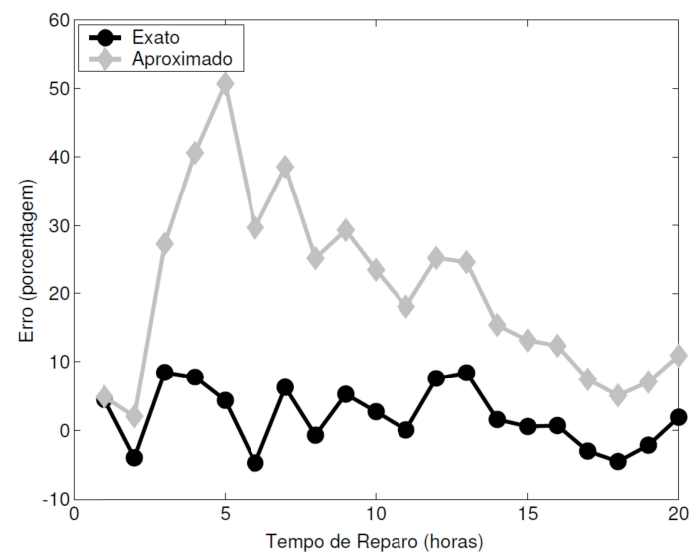

Figura 2: Modelo de Markov simplificado
Em geral, a taxa de transição do estado NORMAL para o estado falhado $\left(\lambda_{15}\right)$, não é observável diretamente, uma vez que os defeitos internos à proteção são invisíveis ao operador (Siqueira, 1999). Em consequência, a taxa de falha de proteção de linhas de transmissão $\left(\lambda_{15}=\lambda_{f}\right)$, principal variável da confiabilidade do sistema de proteção, terá de ser calculada através de outras variáveis observáveis, usando as equações do modelo. As variáveis observáveis são ocorrências e durações de eventos visíveis, tais como: frequências de recusas de atuação e atuações indevidas e os tempos médios de teste e de reparo. Estes parâmetros foram calculados em estudo realizado por Siqueira (1999) para um período de observação de onze anos. Portanto, a taxa de falha do sistema de proteção é determinada por:

$$
\lambda_{f}=\frac{\left(F_{r}+F_{e}\right)}{1-F_{t} \cdot m-r \cdot\left(F_{r}+F_{e}\right)}
$$

onde:

$F_{r}$ é a frequência de recusas de atuação;

$F_{e}$ é a frequência de atuações indevidas;

$m=1 / \mu_{21}$ tempo médio de teste;

$r=1 / \mu_{51}$ tempo médio de reparo;

$F_{t}$ é a periodicidade da manutenção.

Em (2), todos os elementos do lado direito podem ser avaliados por estatísticas dos dados históricos de desligamentos forçados. $F_{r}$ e $F_{e}$ representam, respectivamente, as frequências observadas de recusas e atuações indevidas no período, ambas observáveis, após um tempo longo de adoção da frequência de teste $F_{t}$. Com exceção destas frequências, os demais dados são característicos do sistema de proteção, e independentes da frequência de testes $F_{t}$. Determinam-se as probabilidades em regime permanente dos estados normal, teste e falha através da matriz de transição do diagrama de estados (Billinton \& Allan, 1992) ilustrado na Figura 2:

$$
\begin{aligned}
& {\left[\begin{array}{c}
P_{1} \\
P_{2} \\
P_{5}
\end{array}\right]^{T}\left[\begin{array}{ccc}
1-\lambda_{12}-\lambda_{f} & \lambda_{12} & \lambda_{f} \\
\mu_{21} & 1-\mu_{21} & 0 \\
\mu_{51} & 0 & 1-\mu_{51}
\end{array}\right]=\left[\begin{array}{c}
P_{1} \\
P_{2} \\
P_{5}
\end{array}\right]^{T}} \\
& P_{1}+P_{2}+P_{5}=1
\end{aligned}
$$

A partir de (3) e (4) tem-se : 


$$
\begin{gathered}
P_{1}=\frac{1-F_{t} \cdot m}{1+\lambda_{f} \cdot r} \\
P_{2}=F_{t} \cdot m \\
P_{5}=\frac{\lambda_{f} \cdot\left(1-F_{t} \cdot m\right) \cdot r}{1+\lambda_{f} \cdot r}
\end{gathered}
$$

Finalmente, deve-se mencionar que neste artigo foi considerado que as frequências de atuações indevidas $\left(F_{e}\right)$ e de recusas $\left(F_{r}\right)$ de atuação são correlacionadas com os intervalos de manutenção $\left(F_{t}^{-1}\right)$ através de:

$$
\begin{aligned}
& F_{e}=F_{e}^{0} e^{\delta_{e} / F_{t}} \\
& F_{r}=F_{r}^{0} e^{\delta_{r} / F_{t}}
\end{aligned}
$$

onde:

$F_{e}^{0}\left(F_{r}^{0}\right)$ e $\delta_{e}\left(\delta_{r}\right)$ são o valor inicial e o fator de crescimento, respectivamente, associados com a frequência de atuações indevidas (recusas de atuação).

Neste artigo considera-se que a indisponibilidades das linhas de transmissão são causadas apenas pelas falhas e ações de manutenção nos seus equipamentos de proteção. Contudo, pode-se expandir o modelo Markoviano deste artigo para incluir falhas de outra natureza.

\section{ESTIMAÇÃO DA PV USANDO O MO- DELO MARKOVIANO PROPOSTO}

Nesta seção são apresentados os métodos analíticos usados na estimação do valor esperado da PV. Em 3.1 descreve-se uma técnica inspirada no cálculo de custos de interrupção (Billinton \& Allan, 1996) que é denominada de Método Analítico Aproximado. Em 3.2 descreve-se uma técnica mais precisa que relaxa algumas aproximações usadas no modelo convencional. Esta técnica é denominada Método Analítico Exato.

\subsection{MÉTODO ANALÍTICO APROXIMADO}

A PV está associada com duas componentes: desligamentos programados e não programados. Ambas as componentes podem ser estimadas considerando-se que:

i) a duração de todos os desligamentos é igual a duração média dos desligamentos. ii) O número de desligamentos é igual ao valor esperado da frequência dos desligamentos.

iii) Os desligamentos programados são associados com a duração e frequência do estado 2 na Figura 2.

iv) Os desligamentos não-programados estão associados com a duração e a frequência do estado 5 na Figura 2.

As considerações (i)-(iv) são válidas somente quando há pouca dispersão em torno dos valores esperados das durações dos desligamentos. Caso contrário, as penalidades devem ser estimadas usando-se o Método de Simulação Monte Carlo (Billinton \& Allan, 1992; Billinton \& Li, 1994; Billinton \& Allan, 1996).

A partir das considerações (i), (ii) e (iii) é possível estimar o valor esperado da parcela da PV associada com desligamentos programados $(E[P V p])$ da forma:

$$
\begin{aligned}
E\left[P V^{p}\right] & =\frac{P B}{1440 \cdot D}\left(K_{p} \cdot F_{2} \cdot D_{2} \cdot 1440 \cdot D\right) \\
& =P B \cdot K_{p} \cdot F_{2} \cdot D_{2} \\
& =P B \cdot K_{p} \cdot F_{t} \cdot m
\end{aligned}
$$

onde $D_{2}$ e $F_{2}$ são a duração (em meses) e a frequência (em ocorrências por mês) do estado 2 na Figura 2.

Por outro lado, o valor esperado da PV referente aos desligamentos não-programados $(E[P V o])$ é calculado usandose as considerações (i), (ii) e (iv). Desta forma, tem-se que $E[P V o]$ é:

a) para $D_{5} \leq 300 \mathrm{~min}$.

$$
\begin{aligned}
E\left[P V^{o}\right] & =\frac{P B}{1440 D}\left(K_{o} \cdot F_{5} \cdot D_{5} \cdot 1440 D\right) \\
& =\frac{P B}{1440 D}\left(K_{o} \cdot F_{5} \cdot r \cdot 1440 D\right)
\end{aligned}
$$

b) para $D_{5}>300 \mathrm{~min}$.

$$
\begin{aligned}
E\left[P V^{o}\right] & =\frac{P B}{1440 D} F_{5}\left[300 \cdot K_{o}+K_{p}\left(1440 \cdot D \cdot D_{5}-300\right)\right] \\
& =\frac{P B}{1440 D} F_{5}\left[300 . K_{o}+K_{p}(1440 \cdot D \cdot r-300)\right]
\end{aligned}
$$

onde:

$D_{5}$ e $F_{5}$ são a duração (em meses) e a frequência (em ocorrências por mês) do estado 5 na Figura 2,

$F_{5}=P_{5} r^{-1}=\frac{\lambda_{f}\left(1-F_{t} m\right)}{1+\lambda_{f} r}$ 
O valor esperado da PV $(E[P V])$ é:

$$
E[P V]=E[P V p]+E[P V o]
$$

Neste ponto, deve-se mencionar que os princípios usados para calcular o $E[P V]$, através de (13), são os mesmos aplicados na estimação dos custos de interrupção do fornecimento de energia em redes de distribuição (Billinton \& Allan, 1996). Os custos de interrupção também podem ser estimados usando-se os valores esperados das frequências de falhas e dos tempos de restauração nos pontos de carga. Devido a isto, as estimativas do $E[P V]$ e dos custos de interrupção estão sujeitos à mesma restrição: as variações em torno dos tempos médios de restauração e de desligamentos (programados e não-programados) devem ser pequenas.

\subsection{MÉTODO ANALÍTICO EXATO}

Os valores esperados da PV associados com desligamentos programados e não programados podem ser definidos como:

$$
\begin{aligned}
& E\left[P V^{p}\right]=\frac{P B}{1440 D} E\left[\sum_{i=1}^{N P} g\left(D D P_{i}\right)\right] \\
& E\left[P V^{o}\right]=\frac{P B}{1440 D} E\left[\sum_{i=1}^{N O} h\left(D O D_{i}\right)\right]
\end{aligned}
$$

Os valores esperados mostrados em (14) e (15) são na verdade valores esperados de uma soma de variáveis aleatórias, ou seja (Miller \& Childers, 2004):

$$
E[S]=E\left[\sum_{i=1}^{N} X_{i}\right]=\mu_{x} E[N]
$$

onde:

$\mu_{x}$ é o valor esperado de $X_{i}$;

$E[N]$ é o valor esperado da variável aleatória inteira $N$.

$X_{i} \operatorname{com} i=1, \ldots, N$ é conjunto de variáveis aleatórias com distribuições idênticas.

A partir de (16) tem-se que $E[P V p]$

e $E[P V o]$

são:

$$
E\left[P V^{p}\right]=\frac{P B}{1440 D} E[N P] E\left[g\left(D D P_{i}\right)\right]
$$

$$
E\left[P V^{o}\right]=\frac{P B}{1440 D} E[N O] E\left[h\left(D O D_{i}\right)\right]
$$

Os números esperados de desligamentos programados e não programados podem ser obtidos calculando-se as frequências dos estados de teste e falha do modelo de Markov da Figura 2:

$$
\begin{gathered}
E[N P]=F_{2}=\mu_{21} P_{2}=F_{t} \\
E[N O]=F_{5}=\mu_{51} P_{5}=\frac{\lambda_{f}\left(1-F_{t} m\right)}{1+\lambda_{f} r}
\end{gathered}
$$

Por outro lado, os valores esperados das funções $g\left(D D P_{i}\right)$ e $h\left(D O D_{i}\right)$ estão associados com o cálculo do valor esperado de funções de variáveis aleatórias:

$E[\rho(x)]=\int_{-\infty}^{+\infty} \rho(x) f_{X}(x) d x$

onde: $f_{X}(x)$ é a função densidade de probabilidade da variável aleatória x e $\rho(x)$ é a função da variável aleatória $x$.

Consequentemente, para estimar os valores esperados das funções $g\left(D D P_{i}\right)$ e $h\left(D O D_{i}\right)$ deve-se conhecer as funções densidade de probabilidade associadas com as durações dos estados de falha e teste. Contudo, em uma cadeia de Markov homogênea, o tempo gasto em um estado tem distribuição exponencial com taxa de falha igual a soma de todas as taxas de transição que saem deste estado (Gubner, 2006). Devido $\mathrm{a}$ isto, tem-se que as funções densidade de probabilidade dos estados de falha (estado 5) e teste (estado 2) são dadas por:

$f_{5}(t)=\frac{1}{r} \exp (-t / r)$,

$f_{2}(t)=\frac{1}{m} \exp (-t / m)$

onde $f_{2}(t)$ e $f_{5}(t)$ são as funções densidade de probabilidade associadas, respectivamente, com as durações dos estados 2 e 5 da Figura 2,.

Desta forma, os valores esperados das funções $g\left(D D P_{i}\right)$ e $h\left(D O D_{i}\right)$ são:

$$
\begin{aligned}
E\left[g\left(D D P_{i}\right)\right] & =\int_{0}^{\infty}\left[\frac{K_{p} t}{1440 m D}\right] \exp \left[\frac{-t}{1440 m D}\right] d t \\
& =1440 D K_{p} m
\end{aligned}
$$




$$
\begin{gathered}
E\left[h\left(D O D_{i}\right)\right]=\int_{0}^{300}\left[\frac{K_{o} t}{1440 r D}\right] \exp \left[\frac{-t}{1440 r D}\right] d t+ \\
\int_{300}^{\infty}\left\{\frac{\left[300 K_{o}+K_{p}(t-300)\right]}{1440 r D}\right\} \exp \left[\frac{-t}{1440 r D}\right] d t
\end{gathered}
$$$$
E\left[h\left(D O D_{i}\right)\right]=1440 r D\left[K_{o}+\left(K_{p}-K_{o}\right) \exp \left(\frac{-5}{24 r D}\right)\right]
$$

Substituindo-se (19) e (21) em (17) encontra-se que a penalidade esperada associada com os desligamentos programados é:

$$
E\left[P V^{p}\right]=P B K_{p} F_{t} m
$$

Substituindo-se (20) e (22) em (18) tem-se que a penalidade esperada associada com os desligamentos não-programados é:

$$
E\left[P V^{o}\right]=P B F_{5} r\left[K_{o}+\left(K_{p}-K_{o}\right) \exp \left(\frac{-5}{24 r D}\right)\right]
$$

Finalmente, tem-se a expressão para a estimação do $E[P V]$

$$
\begin{gathered}
E[P V]=E\left[P V^{p}\right]+E\left[P V^{o}\right] \\
=P B F_{5} r\left[K_{o}+\left(K_{p}-K_{o}\right) \exp \left(\frac{-5}{24 r D}\right)\right]+ \\
P B K_{p} F_{t} m
\end{gathered}
$$

É importante observar que as expressões de $E[P V p]$

obtidas pelos métodos analíticos aproximado (10) e exato (23) são idênticas. As expressões de $E[P V o]$

geradas pelos métodos aproximado (11) e (12) e exato (24) são diferentes. Esta diferença é devido ao fato de que o método analítico aproximado estima $E[P V o]$

considerando que o valor esperado da função $\rho(\mathrm{x})$ de uma variável aleatória $x$ é igual a $\rho\left(\mu_{X}\right)$, onde $\mu_{X}$ é o valor esperado da variável aleatória. Esta aproximação não é válida para funções lineares por partes, tais como a parcela da PV devido aos desligamentos não programados (ver (22) ).

\section{MODELOS DE OTIMIZAÇÃO USADOS PARA DETERMINAR O INTERVALO DE MANUTENÇÃO}

Nesta seção são apresentados dois modelos de otimização usados para determinar os intervalos de manutenção das pro- teções das linhas de transmissão. O primeiro modelo se baseia na maximização da disponibilidade. $\mathrm{O}$ segundo modelo, que é uma das contribuições deste artigo, determina o intervalo de manutenção através da minimização do valor esperado da $\mathrm{PV}$.

\subsection{MODELO BASEADO NA MAXIMIZA- ÇÃO DA DISPONIBILIDADE}

Geralmente os intervalos de manutenção são otimizados visando maximizar a disponibilidade de um equipamento (Anders, 1990). No caso do modelo Markoviano da Figura 2, a maximização da disponibilidade está associada com o problema de otimização:

$$
\text { maximizar } \quad P_{1}=\frac{1-\left(F_{t} m\right)}{1+\lambda_{f} r}
$$

onde:

$\lambda_{f}=\frac{\left(F_{r}^{0} e^{\delta_{r} / F_{t}}+F_{e}^{0} e^{\delta_{e} / F_{t}}\right)}{1-F_{t} m-r\left(F_{r}^{0} e^{\delta_{r} / F_{t}}+F_{e}^{0} e^{\delta_{e} / F_{t}}\right)}$

Existe apenas uma variável de decisão no problema de otimização: a frequência de manutenções programadas $F_{t}$ (o inverso do intervalo de manutenção). Não é possível obter uma solução algébrica para o problema (26) a partir das condições de optimalidade, ou seja: $\frac{d P_{1}}{d F_{t}}=0$. Esta restrição é devido a presença dos termos exponenciais associados com $\lambda_{f}$. Apesar disso, é possível resolver (26) usando-se métodos de otimização irrestrita, tais como: Busca Dicotômica, Busca de Fibonacci, Busca Áurea, Método de Interpolação Quadrática e Método de Interpolação Cúbica (Antoniou \& Lu, 2007). Nestes métodos, a solução ótima é obtida baseandose somente no cálculo da função objetivo. Entretanto, não há garantia de que uma solução ótima de (26) seja um mínimo local do $E[P V]$. Em outras palavras, não há garantia de que a maximização de $P_{1}$ seja um problema de otimização equivalente ("proxy") a minimização do $E[P V]$.

\subsection{MODELO BASEADO NA MINIMIZA- ÇÃO DO VALOR ESPERADO DA PV}

No panorama atual do setor elétrico nacional é mais adequado para as empresas de transmissão minimizar o $E[P V]$ do que maximizar a disponibilidade dos equipamentos. Este objetivo pode ser alcançado através da solução do problema de otimização:

$$
\text { minimizar } \quad E[P V]=E[P V p]+E[P V o]
$$

onde: 


$$
\begin{aligned}
& E\left[P V^{p}\right]=\quad P B K_{p} F_{t} m \quad E\left[P V^{o}\right] \\
& P B F_{5} r\left[K_{o}+\left(K_{p}-K_{o}\right) \exp \left(\frac{-5}{24 r D}\right)\right] \\
& F_{5}=\frac{\lambda_{f}\left(1-F_{t} m\right)}{1+\lambda_{f} r} \\
& \lambda_{f}=\frac{\left(F_{r}^{0} e^{\delta_{r} / F_{t}}+F_{e}^{0} e^{\delta_{e} / F_{t}}\right)}{1-F_{t} m-r\left(F_{r}^{0} e^{\delta_{r} / F_{t}}+F_{e}^{0} e^{\delta_{e} / F_{t}}\right)}
\end{aligned}
$$

O problema de otimização (27) tem as seguintes características de otimização em comum com (26): i) ambos são problemas de otimização não-linear irrestrita; ii) a única variável de decisão é a frequência de manutenções programadas $F_{t}$; iii) não é possível obter soluções algébricas para os dois problemas devido aos termos exponenciais associados $\operatorname{com} \lambda_{f}$. Há termos exponenciais na expressão de $E[P V o]$. iv) a solução de (27) pode ser obtida aplicando-se os mesmos métodos usados para resolver (26).

Ao contrário da solução de (26), é possível garantir que uma solução ótima de (27) seja um mínimo local da PV.

\section{RESULTADOS}

Nesta seção são apresentados os resultados obtidos com a aplicação dos modelos e técnicas propostos para estimar e otimizar o valor esperado da PV. Os resultados apresentados nas próximas subseções são organizados como:

i) Em 5.1 apresenta-se um estudo comparativo entre os métodos analíticos aproximado e exato para a estimação do valor esperado da PV; ii) Os intervalos de manutenção obtidos com os modelos de otimização descritos na Seção 4 são mostrados em 5.2. Além disso, são mostradas diversas análises de sensibilidade do valor esperado da PV com relação ao intervalo de manutenção.

\subsection{ESTIMAÇÃO DO VALOR ESPERADO DA PV}

Nesta seção é apresentada uma análise comparativa dos métodos analíticos usados para estimar o valor esperado da PV, isto é: os métodos aproximado e exato descritos nas Subseções 3.1 e 3.2 , respectivamente. As estimativas do $E[P V]$ geradas por estes dois métodos serão comparadas com aquelas obtidas com a Simulação Monte Carlo (Billinton \& Allan, 1992; Billinton \& Li, 1994; Billinton \& Allan, 1996). A Simulação Monte Carlo (SMC) é utilizada como parâmetro para avaliar a precisão das estimativas geradas pelos dois métodos analíticos, ou seja: o método analítico que produzir as estimativas mais próximas daquelas obtidas com a simulação será considerado o mais preciso. Esta comparação é realizada considerando-se que:

i) o tempo médio de duração do teste $(m)$ é igual a 21,7490 horas $\left(3,0207 \times 10^{-2}\right.$ meses $)$; ii) o fator de crescimento $\left(\delta_{e}\right)$ e o valor inicial $\left(F_{e}^{0}\right)$ da frequência de atuações indevidas são iguais a 0,01 e 0,03445 , respectivamente; iii) o fator de cres- cimento $\left(\delta_{r}\right)$ e o valor inicial () da frequência de recusas de atuação são iguais a 0,001 e 0,01867, respectivamente; iv) o intervalo de manutenção $\left(F_{t}\right)$ é de 36 meses; v) o tempo médio de reparo $(r)$ é variado de 1 até 20 horas; vi) a RAP é igual a $\mathrm{R} \$ 25.800 .000,00$. Consequentemente, o pagamento base $(P B)$ é igual $\mathrm{R} \$ 2.150 .000,00$. vii) o tamanho da amostra usado para estimar a PV via simulação foi de 10.000 meses.

A estimação da PV está associada com os seguintes aspectos: i) frequência e a duração dos estados de teste e falha; ii) função não-linear da duração do estado de falha. Devido a isto, é mais adequado estimar a PV via SMC considerando o processo cronológico de transição de estados do equipamento. Neste artigo, o processo cronológico de operação do equipamento é simulado usando-se a amostragem de duração de estados (Billinton \& Li, 1994). O sorteio das durações dos estados do equipamento foi realizado considerando-se que as durações têm distribuição exponencial. O algoritmo conceitual para estimar a PV via SMC é mostrado no Apêndice.

Os $E[P V]$ estimados pelos dois métodos analíticos e pela simulação são mostrados na Figura 3. Pode-se concluir que as estimativas do $E[P V]$ geradas pelo método analítico exato são mais próximas daquelas obtidas via simulação Monte Carlo do que as estimativas calculadas pelo método analítico aproximado. Este fato é um indicativo de que o método analítico proposto neste artigo é mais robusto do que os métodos baseados no cálculo dos custos de interrupção para estimar o $E[P V]$. A precisão dos métodos analíticos com relação à simulação pode ser avaliada de forma mais precisa calculando-se os erros relativos das estimativas do $E[P V]$ obtidas com os métodos analíticos com relação aquelas geradas via simulação. O gráfico dos erros relativos entre os métodos analíticos aproximado e exato é mostrado na Figura 4.

O valor máximo do erro associado com o método analítico aproximado é igual a $50.7287 \%$. Por outro lado, o valor máximo do erro associado com o método analítico exato é apenas $8.5239 \%$. Além disso, o erro absoluto médio associado com os métodos analíticos aproximado e exato são iguais a $20.5925 \%$ e $3.9913 \%$, respectivamente. Estes resultados demonstram quantitativamente que o método analítico exato proposto neste artigo é a técnica mais adequada para estimar o valor esperado da PV. É importante enfatizar que a simulação Monte Carlo é um método baseado na amostragem aleatória. Portanto, os resultados gerados pela simulação serão diferentes para cada sorteio de uma nova amostra. Contudo, os resultados ficam restritos a um intervalo definido por um nível de confiança especificado. Este intervalo contém a média populacional do índice estimado pela simulação através de uma média amostral. Consequentemente, espera-se que os valores estimados pelos métodos analíticos estejam conti- 


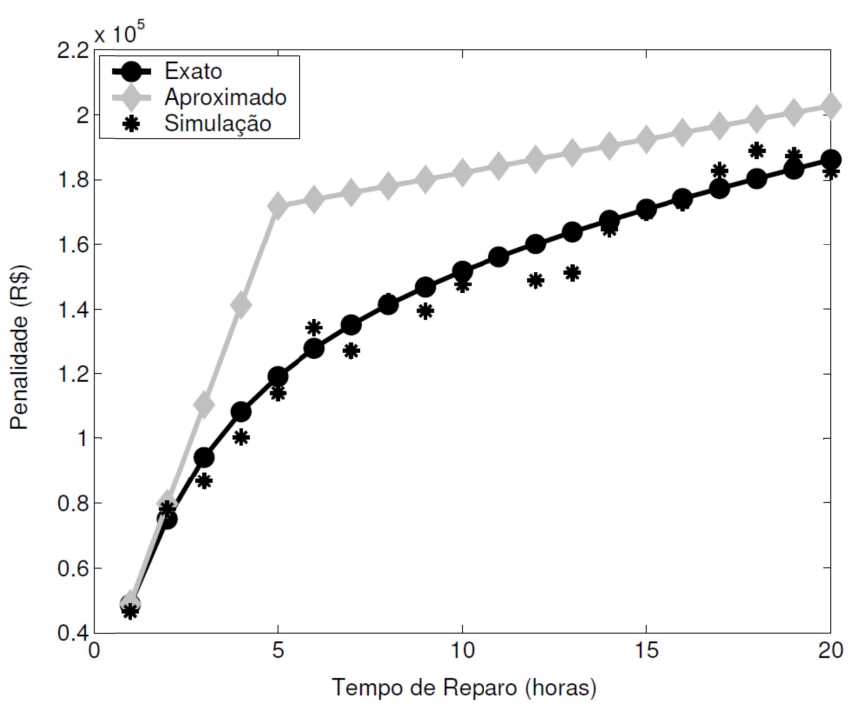

Figura 3: $E[P V]$ calculados pela simulação e pelos métodos analíticos aproximado e exato

dos dentro do intervalo de confiança gerado pela simulação. Caso contrário, o valor esperado estimado por um modelo analítico não estará associado com a média amostral gerada pela simulação, isto é, o modelo analítico não é um bom estimador para o índice probabilístico desejado. Os intervalos de confiança associados com os valores das penalidades estimados via simulação, com o tempo de reparo variando de 1 até 20 horas, são mostrados na Figura 5. Também são mostradas nesta figura as penalidades estimadas pelos métodos aproximado e exato. Pode-se observar que os valores das penalidades estimados via método analítico exato estão contidos dentro dos intervalos de confiança gerados pela simulação. Em outras palavras, o método analítico exato é um bom estimador da média populacional das penalidades. A maior parte das penalidades calculadas pelo método analítico aproximado está fora do intervalo de confiança gerado pela simulação. Desta forma, a simulação não reconhece as estimativas geradas pelo método aproximado como uma média populacional das penalidades.

\subsection{DETERMINAÇÃO DOS INTERVALOS DE MANUTENÇÃO VIA MODELOS DE OTIMIZAÇÃO}

Nesta seção são apresentados os resultados obtidos com os dois modelos de otimização do intervalo de manutenção, ou seja: i) maximização da disponibilidade; ii) minimização do $E[P V]$.

Estes resultados foram obtidos para as mesmas condições definidas na Subseção 5.1. O tempo médio de reparo $(r)$ foi

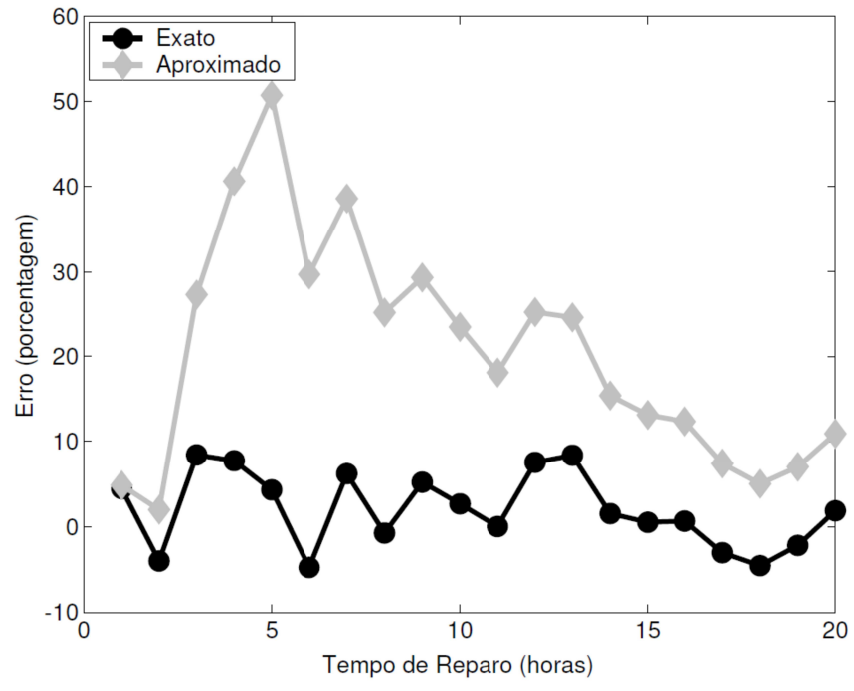

Figura 4: Erros relativos dos métodos analíticos com relação a simulação

fixado em 4,3861 horas $\left(6,0918 \times 10^{-3}\right.$ meses $)$ e a frequência de manutenções programadas $\left(F_{t}\right)$ foi determinada pelos modelos de otimização citados.

Inicialmente, são analisados os efeitos das variações no intervalo de manutenção sobre a disponibilidade (probabilidade do estado normal). Estes efeitos são mostrados na Figura 6. Pode-se observar que a disponibilidade é uma função convexa do intervalo de manutenção no domínio $[1,100]$. Desta forma, é possível calcular o valor ótimo global desta probabilidade no intervalo $[1,100]$. O valor ótimo do intervalo de manutenção e da disponibilidade são iguais a 50,0497 meses e 0,9990 , respectivamente. A maximização da disponibilidade foi realizada através da função fminbnd do toolbox de otimização do MATLAB (Branch, 1996). O método "default" para solução de problemas de otimização irrestrita na função fminbnd é Busca Áurea com interpolação polinomial (Antoniou \& Lu, 2007).

Em seguida, foram analisadas as variações no $E[P V]$ e nas suas componentes ( $E[P V p]$ e $E[P V o])$ com relação ao intervalo de manutenção. As variações de $E[P V p]$ e $E[P V o]$ com relação ao intervalo de manutenção são mostradas na Figura 7. É importante citar que os valores de $E[P V p]$ e $E[P V o]$ mostrados nesta figura foram obtidas usando-se o método analítico exato, pois este método foi identificado como o mais preciso para estimar o $E[P V]$ nos testes realizados na Subseção 5.1.

A partir da Figura 7, Pode-se concluir que $E[P V p]$ aumenta quando o intervalo de manutenção é diminuído. Por outro lado, pode-se observar que $E[P V o]$ é reduzido quando há 


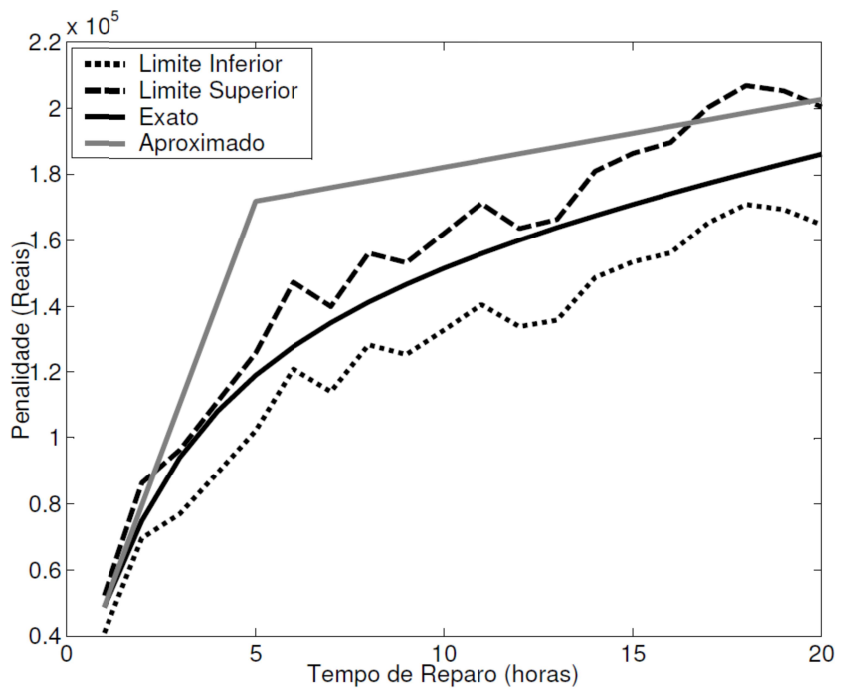

Figura 5: Intervalos de confiança gerados pela simulação e estimativas do $E[P V]$.

um aumento na frequência de manutenções. Além disso, as variações no $E[P V](E[P V p]+E[P V o])$ com relação ao intervalo de manutenção são mostradas na Figura 8 . Verificase que o $E[P V]$ é uma função convexa do intervalo de manutenção no domínio $[1,100]$. Devido a isto, existe um valor ótimo global para o $E[P V]$ no intervalo $[1,100]$. Os valores ótimos do $E[P V]$ e do intervalo de manutenção obtidos com a função fminbnd são iguais a R \$ 60.722,1707 e 24,0145 meses, respectivamente. Estes valores são identificados através das retas tracejadas na Figura 8. É importante observar que o intervalo de manutenção obtido com a maximização da probabilidade do estado normal (reta vertical pontilhada na Figura 8) é maior do que aquele obtido com a minimização do $E[P V]$. Em outras palavras, a maximização da disponibilidade resulta em um intervalo de manutenção subótimo com relação à minimização do $E[P V]$. Por exemplo, o $E[P V]$ obtido através da solução do problema (26) (R $\$$ $93.772,8275)$ é $35,2455 \%$ maior do que o $E[P V]$ associado com o problema (27) $(\mathrm{R} \$ 60.722,1707)$.

Finalmente, é importante avaliar os percentuais associados com as parcelas do $E[P V]$ obtidos com os modelos de maximização da disponibilidade e minimização do $E[P V]$. Estes percentuais são mostrados nas Figuras 9 e 10. Observa-se que a solução ótima do problema de minimização do $E[P V]$ produz uma divisão mais equitativa da $E[P V]$, entre as suas componentes $(E[P V p]$ e $E[P V o])$, do que a maximização da disponibilidade.

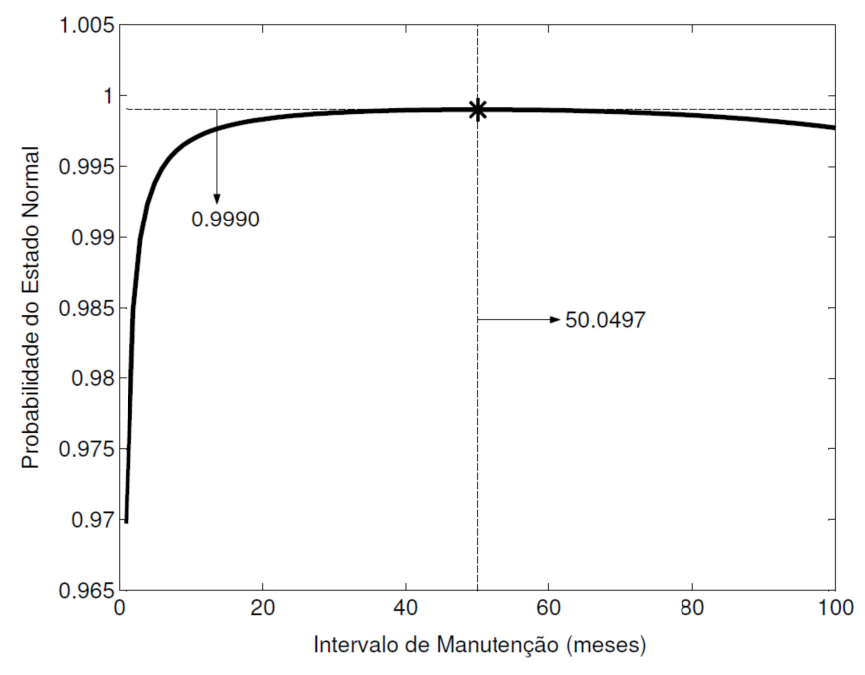

Figura 6: Variação da disponibilidade com relação ao intervalo de manutenção $\left(1 / F_{t}\right)$.

\section{CONCLUSÕES}

Neste artigo foi apresentado um modelo probabilístico para o sistema de proteção de linhas de transmissão que incorpora o efeito dos intervalos de manutenção nas taxas de falha do modelo. Os modelos probabilísticos do sistema de proteção foram usados para otimizar os intervalos de manutenção de acordo com dois paradigmas: maximização da disponibilidade do equipamento e minimização do valor esperado da Parcela Variável $(E[P V])$. Os testes realizados demonstraram que o último paradigma é mais adequado à realidade do setor elétrico nacional. Esta adequação é devido ao fato de que as penalidades estimadas com a minimização do $E[P V]$ são menores do que aquelas calculadas com a maximização da disponibilidade dos equipamentos. Em ambos os paradigmas citados, foi possível otimizar os intervalos de manutenção usando-se apenas algoritmos para a solução de problemas de programação não-linear irrestrita unidimensional. Adicionalmente, foi proposto um método analítico exato para estimar o $E[P V]$. Os $E[P V]$ calculados por este método são mais precisos do que aqueles obtidos com aproximações tradicionalmente usadas na estimação probabilística dos custos de interrupção.

Os custos associados com as manutenções programadas não são considerados no método de determinação do intervalo de manutenção proposto neste artigo. Isto é, a periodicidade da manutenção é determinada considerando somente as penalidades devido aos desligamentos programados e não programados. Contudo, os custos de manutenção (mão de obra, substituição de equipamentos, inspeção, etc.) podem ser adicionados diretamente à função objetivo (27) para minimizar 


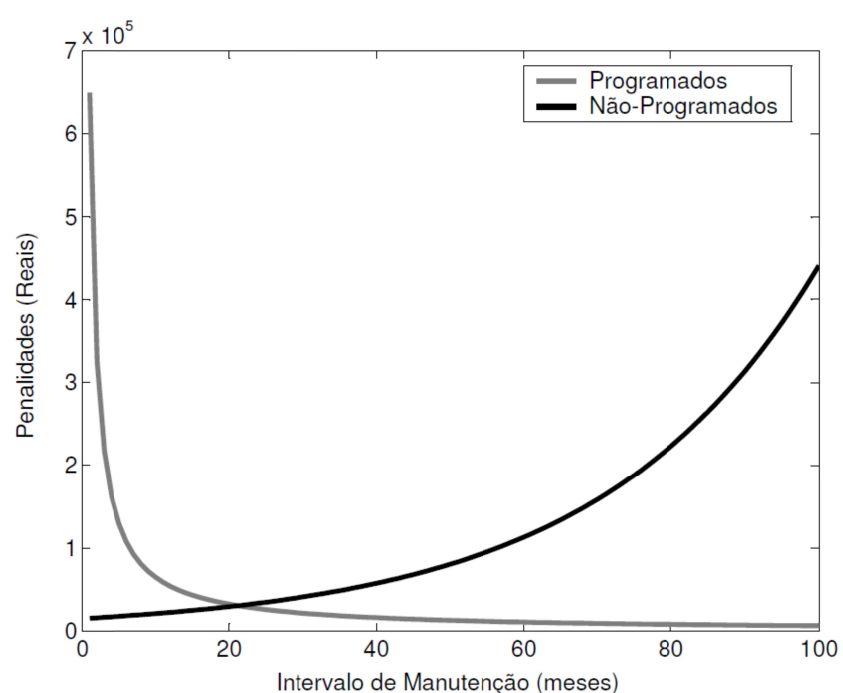

Figura 7: Variações de $E[P V p]$ e $E[P V o]$ com relação ao intervalo de manutenção $\left(1 / F_{t}\right)$.

simultaneamente as penalidades e os custos de manutenção. Também é possível incluir os custos de reparo do equipamento na função objetivo (Endrenyi, 1998). Esta estratégia de modelagem dos custos de manutenção tende a causar um aumento nos intervalos de manutenção, pois a componente associada com os custos de manutenção (frequência de manutenção vezes o custo de manutenção unitário) funciona como um termo de penalidade na função objetivo. Os aspectos discutidos acima serão abordados pelos autores em futuras publicações.

\section{REFERÊNCIAS BIBLIOGRÁFICAS}

Agência Nacional de Energia Elétrica (2007) Resolução Normativa $N^{\circ} 270$ de 26 de junho de 2007 (?) , Disponível de: <http://www. http://www.aneel.gov.br $>$ [Acessado em 22 de março de 2010]

Antoniou, A. \& Lu, W., (2007) Practical Optimization: Algorithms and Engineering Applications. Springer.

Anders, G. J. (1990) Probability Concepts in Electric Power Systems. John Wiley \& Sons.

Billinton, R. \& Allan, R.N. (1992) Reliability Evaluation of Engineering Systems: Concepts and Techiniques. Plenum Press.

Billinton, R. \& Allan, R. N. (1996) Reliability Evaluation of Power Systems. 2nd ed. Plenum Press.

Billinton, R. \& Li, W. (1994) Reliability Assessment of Electric Power Systems Using Monte Carlo Methods. Plenum Press.

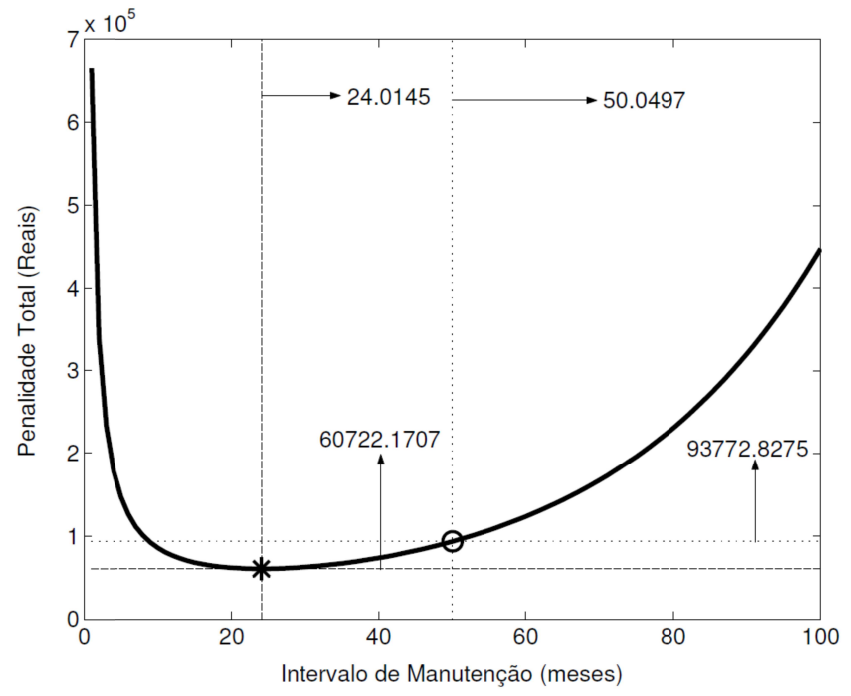

Figura 8: Variação do $E[P V]$ com relação ao intervalo de manutenção $\left(1 / F_{t}\right)$.

Branch, M. A. (1996) MATLAB Optimization Toolbox: User's Guide. Math Works, Inc.

Endrenyi, J., (1978) Reliability Modeling in Electric Power Systems, John Wiley \& Sons.

Endrenyi, J., Anders, G. J., \& Leite da Silva, A. M. (1998), Probabilistic Evaluation of the Effect of Maintenance on Reliability - An Application. IEEE Trans. on Power Systems, 13(2) Maio, pp. 576-583.

Gubner, J. A. (2006) Probability and Random Processes for Electrical and Computer Engineers. Cambridge University Press.

Howard, R.A. (1971) Dynamic Probabilistic Systems - Volume I: Markov Models. John Wiley \& Sons.

Miller, S. L. \& Childers, D. (2004) Probability and Random Processes: with Applications to Signal Processing and Communications. 2nd ed. Academic Press.

Pereira, F. L. P. (2009), Determinação do Intervalo de Manutenção Programada da Proteção de Linhas de Transmissão Considerando-se Penalidades Associadas à Indisponibilidade. Tese de Doutorado, Pontifícia Universidade Católica do Rio de Janeiro.

Siqueira, I. P. (1999) Processos de Decisão Markovianos em Sistemas de Segurança e Proteção. Dissertação de Mestrado, Universidade Federal de Pernambuco. 


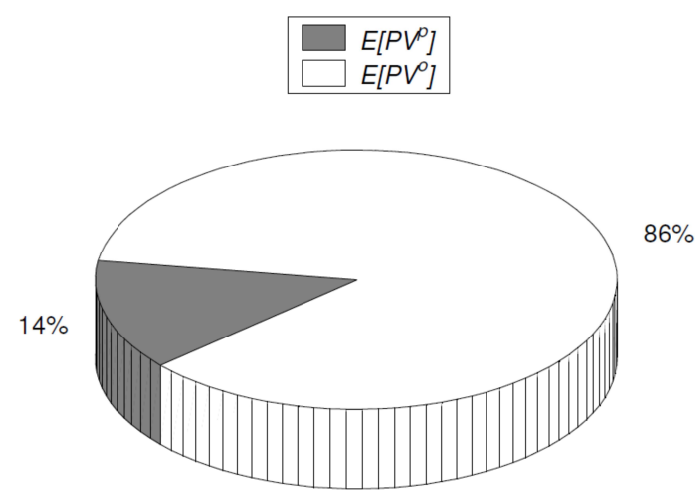

Figura 9: Percentuais associados com $E[P V p]$ e $E[P V o]$ para a maximização as probabilidade do estado normal.

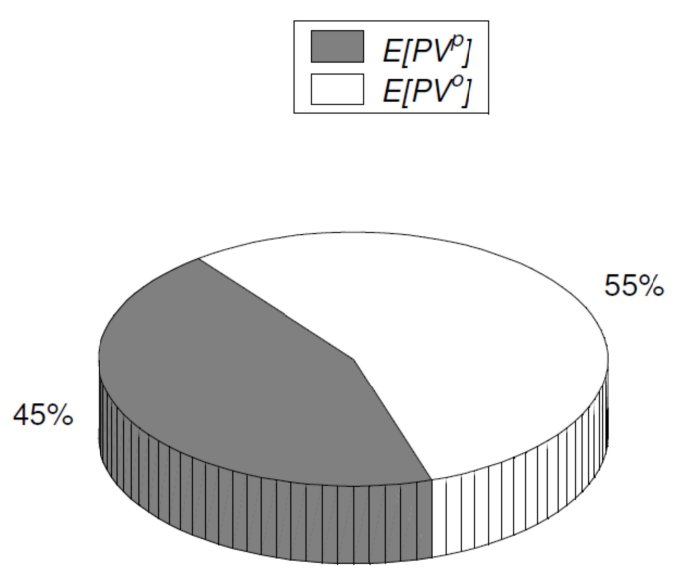

Figura 10: Percentuais associados com $E[P V p]$ e $E[P V o]$ para a maximização as probabilidade do estado normal.

\section{APÊNDICE}

O algoritmo conceitual da SMC usada para estimar a PV é mostrado abaixo:

i)Faça $P V^{e s t}=0$, onde $P V^{e s t}$ é o valor estimado da PV.

ii)Repita os passos (iii)-(ix) para $i=1, \ldots, N S$, onde $i$ é o contador de simulações e $N S$ é tamanho da amostra de meses que serão sorteados (10.000).

iii)Faça $t^{\text {sim }}=0$ e $P V^{\text {sim }}=0$, onde $t^{\text {sim }}$ é o tempo de simulação e $P V^{\text {sim }}$ é o valor da PV para o i-ésimo mês simulado.

iv)Faça $s=0$, onde $s=1,2$ e 5 indica que o equipamento está operando nos estados normal, teste ou falha, respectivamente.
v)Repita os passos (vi)-(viii) enquanto $t^{\text {sim }}<1$ mês.

vi)Sorteio do tempo de residência no estado atual e atualização da PV:

vi-a)Se $s=1$, então $t_{12}=-\left(1 / \lambda_{12}\right) \log \left(Z_{1}\right)$ e $t_{15}=$ - $\left(1 / \lambda_{15}\right) \log \left(Z_{2}\right)$, onde $Z_{1}$ e $Z_{2}$ são dois números aleatórios com distribuição uniforme. Se $\min \left\{t_{12}, t_{15}\right\}=$ $t_{12}$, então $d^{s i m}=t_{12}$ e $s^{\text {new }}=2$, onde $d^{\text {sim }}$ é a duração do estado atual e $s^{\text {new }}$ é o próximo estado de operação do equipamento. Caso contrário $\left(\min \left\{t_{12}, t_{15}\right\}=\right.$ $\left.t_{15}\right), d^{\text {sim }}=t_{15}$ e $\left.s^{\text {new }}=5\right)$.

vi-b)Se $s=2$, então: $d^{\text {sim }}=-\left(1 / \mu_{21}\right) \log \left(Z_{1}\right) ; s^{\text {new }}=1$ e $P V^{s i m}=P V^{s i m}+P B \times K_{p} \times d^{s i m}$

vi-c)Se $s=5$, então: $d^{\text {sim }}=-\left(1 / \mu_{51}\right) \log \left(Z_{1}\right)$; e $s^{\text {new }}=$ 1 e atualize a PV de acordo com a seguinte equação:

$$
P V^{s i m}=\left\{\begin{array}{r}
P V^{s i m}+P B \times K_{o} \times d^{s i m}, \\
\text { para } 1440 \times \mathrm{D} \times \mathrm{d}^{\text {sim }}<300 \mathrm{~min} \\
P V^{\text {sim }}+\left(\frac{P B}{1440 D}\right) \times\left[300 \times K_{o}+\right. \\
\left.K_{p}\left(1440 \times D \times d^{\text {sim }}-300\right)\right], \\
\text { caso contrario }
\end{array}\right.
$$

vii)Atualize o tempo de simulação:

viii)Atualize o estado do equipamento: $s=s^{\text {new }}$

ix)Atualize o valor estimado da PV: $P V^{e s t}=P V^{e s t}+$ $P V^{\text {sim }}$

x)Calcule o valor estimado da PV: $P V^{e s t}=P V^{e s t} / N S$ 